Association for Information Systems

AIS Electronic Library (AISeL)

\title{
A Holistic Framework for Al Systems in Industrial Applications
}

Can Kaymakci

Fraunhofer Institute for Manufacturing Engineering and Automation IPA, Institute for Energy Efficiency in Production EEP, University of Stuttgart

Simon Wenninger

FIM Research Center, University, Project Group Business \& Information Systems Engineering of the Fraunhofer FIT of Applied Sciences Augsburg

Alexander Sauer

Fraunhofer Institute for Manufacturing Engineering and Automation IPA, Institute for Energy Efficiency in Production EEP, University of Stuttgart

Follow this and additional works at: https://aisel.aisnet.org/wi2021

Kaymakci, Can; Wenninger, Simon; and Sauer, Alexander, "A Holistic Framework for Al Systems in Industrial Applications" (2021). Wirtschaftsinformatik 2021 Proceedings. 3.

https://aisel.aisnet.org/wi2021/RDataScience/Track09/3

This material is brought to you by the Wirtschaftsinformatik at AIS Electronic Library (AISeL). It has been accepted for inclusion in Wirtschaftsinformatik 2021 Proceedings by an authorized administrator of AIS Electronic Library (AISeL). For more information, please contact elibrary@aisnet.org. 


\title{
A Holistic Framework for AI Systems in Industrial Applications
}

\author{
Can Kaymakci ${ }^{1,2}$, Simon Wenninger ${ }^{3,4}$, Alexander Sauer ${ }^{1,2}$ \\ ${ }^{1}$ Fraunhofer Institute for Manufacturing Engineering and Automation IPA, Stuttgart, \\ Germany \\ can.kaymakci@ipa.fraunhofer.de, alexander.sauer@ipa.fraunhofer.de \\ ${ }^{2}$ Institute for Energy Efficiency in Production EEP, University of Stuttgart, Stuttgart, \\ Germany \\ ${ }^{3}$ FIM Research Center, University of Applied Sciences Augsburg, Augsburg, Germany \\ simon.wenninger@fim-rc.de \\ ${ }^{4}$ Project Group Business \& Information Systems Engineering of the Fraunhofer FIT, \\ Augsburg, Germany
}

\begin{abstract}
Although several promising use cases for artificial intelligence (AI) for manufacturing companies have been identified, these are not yet widely used. Existing literature covers a variety of frameworks, methods and processes related to AI systems. However, the application of AI systems in manufacturing companies lacks a uniform understanding of components and functionalities as well as a structured process that supports developers and project managers in planning, implementing, and optimizing AI systems. To close this gap, we develop a generic conceptual model of an AI system for the application in manufacturing systems and a four-phase model to guide developers and project managers through the realization of AI systems.
\end{abstract}

Keywords: Manufacturing AI System, Intelligent Agents, Machine Learning.

\section{Introduction}

The digitization and automation of products, plants and manufacturing processes continues to increase and receives new impulses from modern information technology. Companies are constantly forced to adapt to new technologies and remain competitive. Today companies are facing the next big change - artificial intelligence (AI) systems [1]. Due to the relatively new approach of integrating AI into manufacturing systems, by integrating not only preset programs with explicit instructions and programmed control processes, but also knowledge-based on historical data, the acceptance of these systems is not very pronounced. In research AI systems in manufacturing are historically proposed and developed but not yet widely used in practice [2].

Especially in manufacturing, numerous use cases have been identified where AIcontrolled applications for pattern recognition, process automation, computer vision, nonlinear control, robotics, data mining or process control systems can be used and existing solutions can be made more efficient and effective or even enable solutions at 
all [4]. Thus, company goals such as cost reduction or quality improvement can be supported to remain competitive. Nevertheless, compared to big tech companies like Google, Facebook or Microsoft, the manufacturing industry still has problems integrating AI-driven approaches to optimize and automate manufacturing processes [5].

The manufacturing industry is the backbone of today's economy. In order to remain competitive, the manufacturing industry began early to experiment with AI applications (predictive maintenance, quality control and demand planning) [6]. Despite many successful individual AI experiments and use case, implementations in industry, which concentrate on individual details, a holistic concept for the planning, implementation, and optimization of AI systems, is missing.

A conceptual modeling of AI-based information systems enables the use of AI as well as the continuous improvement of the models. Additionally, a conceptual model that supports industrial companies in the introduction and implementation of AI systems to increase the dissemination of the systems, is needed. To close this gap the following two research questions (RQs) are formulated:

Which components are part of an AI system and how can an AI system be defined generically in a conceptual model?

How should a generic process for planning, implementation, and optimization of AI systems be structured?

We address the RQs by defining and describing an AI system with a conceptual model and developing a generic process to plan, implement and optimize an AI system following a design science research approach. With the aim of laying the foundation of a generic approach of modelling AI systems, we contribute by first deriving the necessity of a holistic concept modeling an AI system in the manufacturing industry by identifying the requirements of intelligent systems used in manufacturing. Second, we propose a set of components to model an AI system. The specific attributes and the relationships between the components are developed. Third, we define a process model to plan, implement and optimize the modeled AI system into existing manufacturing and information system environments considering the specific requirements in manufacturing such as maintaining process stability.

This article is structured in eight sections. The second section attempts to define AI systems and the motivation for manufacturing companies of the described concept are elaborated by analyzing existing literature and comparing state-of-the-art processes/concepts. Second, in Section 3 we introduce our methodological approach before our conceptual model for an AI system with its components is described (Section 4) and a phase model for developing AI systems is introduced in a third step (Section 5). In Section 6 we demonstrate our designed artifacts with a use case of an anomaly detection in energy consumption for a German metal processing company. We discuss and validate the design artifacts with findings from the use case and expert interviews in Section 7 before we conclude in the final Section 8 . 


\section{Why the Manufacturing Industry Needs a Holistic Concept}

The continuous improvement and optimization of processes is one of the key requirements of manufacturing systems [7]. Therefore, AI techniques, especially machine learning, are applicable for realizing intelligent systems [2]. The terminology of AI is difficult to define clearly, but its most common interpretation is that of automation of rational behavior [8]. First, AI in manufacturing was used to improve quality, especially in the semiconductor industry [3]. In recent years, with the advent of large amounts of data, intelligent sensors and improved computing power, AI has also been used in other areas such as process control, demand planning or logistics [1]. Nevertheless, the introduction of AI systems still poses major challenges for manufacturing companies, especially data quality, data processing, model selection or cyber security issues [5]. In research and practice many attempts have already been made to develop frameworks and tools to address these challenges. The published approaches, which are not limited to application in manufacturing systems, can be divided into two segments, whereby the distinction is partly fluent. One segment focuses on the generic description of AI systems by means of a conceptual model that encompasses features, functions, or the underlying components of AI systems. The second segment deals with processes for the introduction, development, or operation of AI systems. The focus is not necessarily on AI systems, but rather on machine learning applications.

The research on conceptual models shows heterogeneous results regarding the definition of AI systems, their functionalities and components, and the structure for their description. Nalchigar et al. [9] present a conceptual modeling framework for designing business analytics systems by determining the requirements of the analytical solution. They introduce the layers business, analytics, and data preparation. Another approach to define a holistic industrial AI ecosystem, where different technologies are categorized into operation, platform, analytic and data technology, is depicted by Lee et al. [2]. Their industrial AI ecosystem focuses on building and integrating AI systems in existing information systems. Wang et al. [10] create an architecture for selforganized multi-agent systems for smart factories. The specific architecture focuses on the interoperability between different machines, information systems and other data sources. Van den Heuvel and Tamburri [11] divide an AI system into three different layers - data, intelligence and application. In contrast, Simard et al. [12] present a nontechnical approach to design AI systems by differentiating between machine learning and machine teaching. Whereas "machine learning" focuses more on implementing the appropriate algorithm and model, "machine teaching” specifies the steps (e.g. labeling, feature engineering, schema definition) for teaching the model by using domain knowledge. It can be observed that there is no uniform understanding of the subject of AI systems in literature. Sculley et al. [13] postulate a lack of an abstract description of AI systems, since there is no abstraction to support AI systems compared to the modeling of relational databases. Much more, a generic, understandable, and applicable definition and description of AI systems is necessary. The listed approaches and frameworks set different priorities, such as the integration of AI systems into an existing ecosystem of information systems, without specifying the AI system itself. In addition, 
the description of the central component of the agent, which we will introduce in section 4 , is missing in the specifications. The variety of different frameworks and perceptions of AI systems makes it difficult to deploy AI systems in a targeted and value adding way for companies that have not yet acquired comprehensive knowledge about the application of AI systems in their production systems.

The second of literature segment investigating processes for the introduction, development, or operation of AI systems is even more diverse. Next to the classic data mining approaches like Knowledge Discovery in Databases (KDD) [14], Cross Industry Standard Process for Data Mining (CRISP-DM) [15] or Team Data Science Process (TDSP) [16], which focus on the process of how to gain information or knowledge from large datasets, other workflow and process models related to machine learning have been developed. Transferring the data mining approaches to the operational use of AI systems in industry reveals a weak point. The approaches focus on extracting information from static historical data in order to create a data-driven model and evaluate it without or only partially considering integration and use as an information system that is interoperable with the manufacturing processes themselves. This is particularly important for the practical use of AI systems. In addition, software solutions that support processes such as data understanding or model management are missing [17]. A possible approach to implement AI systems into existing information systems is to extend software engineering and design practices by considering the specifics in machine learning projects [18]. Amershi et al. [19] elaborates the differences between traditional and AI-based software engineering. Kessler et al. [20] introduce a holistic machine learning lifecycle process from business understanding to model optimization and maintenance as well as implications for enterprise data management based on the type of data, the different roles in machine learning groups and the life cycle of machine learning models. Moreb et al. [21] show a framework for the technical implementation on a coding level for machine learning applications in health information system to enhance the systems efficiency. Kriens and Verbelen [22] present current techniques and methods to manage complex AI-based software. They postulate to package AI models to capture all necessary metadata for automating the deployment process. Lwakatare et al. [23] analyzed software-intensive systems with ML components in industrial settings and derived five different evolution stages of AI systems - prototyping, non-critical deployment, critical deployment, cascading (more than one model) deployment and Autonomous Systems in a case study. The comparison of the different approaches and processes shows that, despite their generic character, the processes mostly provide no precise guidelines for actual implementation in practice or only for individual phases in the AI system life cycle. Several challenges have been described in literature supporting this finding. Chen et al. [24] define four specific challenges in building production ready AI systems. First, the multitude of different tools and frameworks available for the development of AI systems makes it almost impossible to know all tools and frameworks. Second, most of the available tools do not have a particular experiment tracking implemented. Third, the reproducibility of AI systems is a major challenge when transferring models into production. Last, deploying models can be challenging with regard to training and inference. Sculley et al. [13] conclude that modeling, learning and predicting with a 
data-driven model represents only the smallest part in building an AI system. They found that aspects such as configuration, data acquisition or monitoring are more important than the ready-to-use models from research. To avoid pipeline jungles or dead experimental code paths, the entire life cycle of machine learning must be planned [13]. To solve the mentioned challenges further software packages and platforms were developed by Google [25], Uber and Chen et al. [24]. Nevertheless, to date, it is necessary to stack different tools and frameworks together to get an end-to-end solution for an AI system that supports manufacturing processes.

Summarizing, the problem is not the availability and maturity of the technology and AI solutions themselves, but rather that each company has its own individual systems and characteristics as well as no uniform understanding of AI systems. Hence, there is a need for an abstract definition of an AI system within a conceptual model. Missing methods, processes, and frameworks available in the literature guiding engineers, developers, and project managers in manufacturing companies in the planning, implementation, and optimization of AI systems require a generic end-to-end process based on the conceptual model of AI systems. The transfer of classical procedures of systems engineering and software development to AI systems is insufficient and does not cover the specifics of data-driven approaches [18, 19]. For manufacturing companies to withstand increasing cost and efficiency pressure, a uniform procedure for the introduction and operation of AI systems is necessary, which can be individually adapted to the company.

\section{$3 \quad$ Methodology and Study Design}

We organized research in this paper by the design science in information systems research framework combining behavioral science and design science paradigms [26]. In terms of Gregor and Hevner [27] we contribute an improvement to existing research and solutions. We argue that even though the application domain of AI in industrial applications is relatively high, the solution maturity is low, as we are among the first to define a generic model for AI systems and develop a structured process for its implementation. In a first step we derived requirements from literature conducting a semi-structured literature search in the databases Google Scholar, ScienceDirect, Scopus, Semantic Scholar, and AIS eLibrary with the keywords "AI in manufacturing", "AI system", "software engineering" as well as expert interviews (employees in manufacturing companies, AI research experts), whose results we presented in Section 2. The acceptance criteria extracted from expert interviews are completeness of the artifacts, their easy understanding and traceability. After several iterations of designing our artifacts we demonstrated our AI system and applied our generic process with a use case of AI-based anomaly detection in energy consumption (see Section 6) and evaluated the final artifacts again with expert interviews (Section 7). We communicate our results with this paper enabling practitioners to integrate AI in manufacturing systems and industrial applications. 


\section{$4 \quad$ Developing an AI system for Industrial Process Optimization - a Generic System Concept}

\subsection{AI System}

To build an AI system aligned with existing manufacturing processes and information systems a conceptual model of the AI system is necessary. The conceptual model is the interface to the actual requirements of an AI system and can be seen as an abstract, formal description of the AI system [28]. The theoretical foundations on conceptual modeling of information systems are based on the framework presented by Wand et al. [28]. A conceptual model of an AI system structures its underlying components and systems with the purpose of enabling a fast scalability [29]. The conceptual modeling of a system, also called ontological modeling, is an integral part of the implementationindependent conception phase in database design and is regarded as an orientation here [30]. The basic goal of the presented AI system is the beneficial and value-adding transformation (data processing) of incoming data from data sources to outgoing data in the form of actions of an agent. Due to the modular structure of the AI system the conceptual model covers central components and related functionalities. First, physical objects such as sensors or information systems like ERP systems are data sources that can push and pull data to and from other data sources. Components with a data input, data processing and data output functionality are called data processing unit (DPU). Two specific DPUs need to be considered in depth - the data-driven model and the agent.

An AI system consists of at least DPUs, a data-driven model and an agent, which takes predictions of data-driven models as an input and processes it to an action for the subsequent systems or objects. The components of AI systems and their interaction are visualized in Figure 1. Related to the concept of Russell et al. [8], agents provide a specific benefit manifested as action for the manufacturing system and the associated subsystems. In addition, agents can occur in a wide variety of forms to support the manufacturing system. An agent could be a human in the loop, which interprets the outcome of a model and triggers an action.

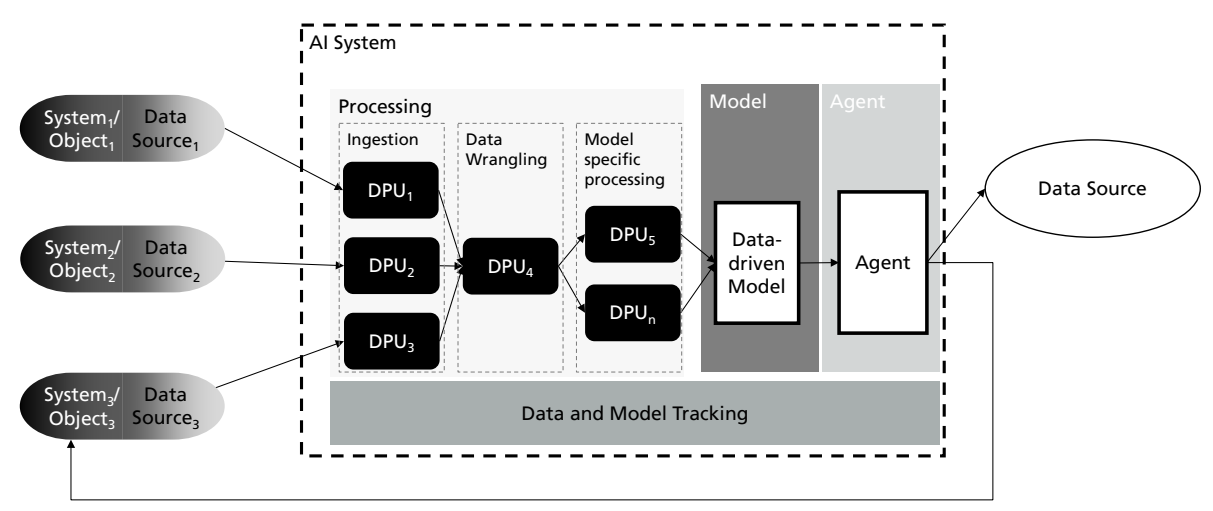

Figure 1. Conceptual model of an AI system 


\subsection{Data Source}

A data source can be a system or an object that sends one or more variables to another system or object at a specified time interval. It is important to mention that data sources not only have to be backed by a physical object but could also be a relational database or a file server - things that can be encapsulated from the real world. A data source could also receive data. A good example is a programmable logic controller (PLC) that receives data from sensors and sends the data to subsequent systems like an ERP system or an energy management system. The metadata of each attribute is stored within the data source (data type, format, etc.). For the planning and design of an AI system, necessary data sources inside and outside of the manufacturing systems must be identified. Domain expertise and experiences from other systems enable identifying relevant data sources from the environment that can influence the result of the AI system [15, 31]. For the individual and exemplary process of predicting machine failure with an AI system, possible data sources such as power consumption, pressure, temperature or speed must be identified. This allows modelling real phenomena such as machine failure or power consumption [32].

\subsection{Data Processing Unit}

A data processing unit (DPU) is defined as a base functional module unit within an AI system that consists of a data input, data transformation and data output module. The transformation module processes the input by explicit and rule-based instructions or specific mathematical functions to a fixed data output. The transformation of a data input into a data output is carried out using various tools and methods. The number of DPUs can be varied as required and adapted to the individual case. Modeling and implementing DPUs can be used for data preprocessing and feature engineering steps [14]. One specific task of a DPU could be the deletion of duplicates, the reconstruction of missing values, scaling input parameters, or the standardization of values. Note, that data-driven models and agents are DPUs with a specific structure and functionality described in the next sections.

\section{Data-Driven Model}

The data-driven model is a DPU with specific characteristics considering the transformation of the data input into an output. The modelling of the relationship between data input and the desired output is done dynamically by optimizing the parameters of a previously defined model with basic assumptions about its form. The challenge here is to choose the right model depending on the requirements of the AI system and to optimize the parameters of the model. In practice a lot of data-driven models are related to machine learning algorithms such as decision trees or neural networks. The optimization of the parameters is also often referred to as "inductive learning as a process of finding a hypothesis that agrees with the observed examples" [8]. The parameters are optimized by training the model with the data input. Some datadriven models additionally use the model output as a label for the optimization of the parameters (e.g. supervised learning). Especially, when traditional theory-driven 
approaches for modeling real phenomena are too complex, data-driven approaches are particularly suitable. Data-driven approaches do not follow the exact and simplified modelling of reality by known and established theories, but consider the input of data sources as "ground truth" [33].

\section{AI Service/Agent}

An agent is defined as a DPU that can consume the output of the data-driven model(s) and transforms or processes the output for further applications. Other approaches such as reinforcement learning agents are also capable of action that do not rely on models [8]. The basic goal of an agent developed for industrial applications is to improve a manufacturing process. Three different cases are to be considered. First, an already existing automated process can be improved with the help of an agent (e.g. temperature regulation through reinforcement learning). Second, a manual process can be enhanced (e.g. recommendation system for energy managers). Third, it is possible to fully automate a manual process (e.g. automation of energy procurement). An agent can also describe the interface to other systems or subsystems. Specifically, an agent could be used by other systems such as the Manufacturing Execution System (MES) or a control system. Especially in control systems of industrial energy supply systems agents can be used to optimize the overall performance of a manufacturing system [34]. The integration of the AI system, or information systems in general, with other information systems is one of the big challenges in developing applications in the manufacturing industry. Therefore, easy to use interfaces must be planned and implemented for the interoperability between those systems [35].

Next to the superordinate cases, agents fulfill different functions, which are manifold described in literature. Hofmann et al. [36] provide an overview and define seven general variants of agents which are relevant for the AI system - Perceiving, Feature extraction and identification, Reasoning, Prediction, Decision, Action and Generation. In line with the problem to be solved by the AI system, the listed functionalities can be used to design the agent for each use case.

\section{$5 \quad$ Phase Model for Developing AI Systems}

\subsection{Developing the Phase Model}

After having defined an AI system contributing to understanding its characteristics, we propose a four-phase model for planning, building, and maintaining an AI systemplanning, experimentation, implementation, and optimization during operation (see Figure 2). We derived our phase model from existing frameworks in literature (see section 2) and from the experience gained in current research projects in setting up several AI systems for industrial applications. In a further step, we validated our work with expert interviews in software engineering and machine learning, which led to minor improvements on the way to the current version. The goal is to present the model as understandable as possible and yet generic. In addition, more detailed frameworks can be integrated into our model in individual phases, which allows us to customize the 
application of our models as desired. Nevertheless, two major points must be considered, which are especially important when building AI systems in a complex information system landscape. The planning phase summarizes all the necessary planning for the AI system, since a corresponding effort must be made to determine the direction for successful further steps. Second, regarding AI systems (especially in manufacturing), it should be noted that a distinction must be made between an experimentation phase and the deployment in production systems.

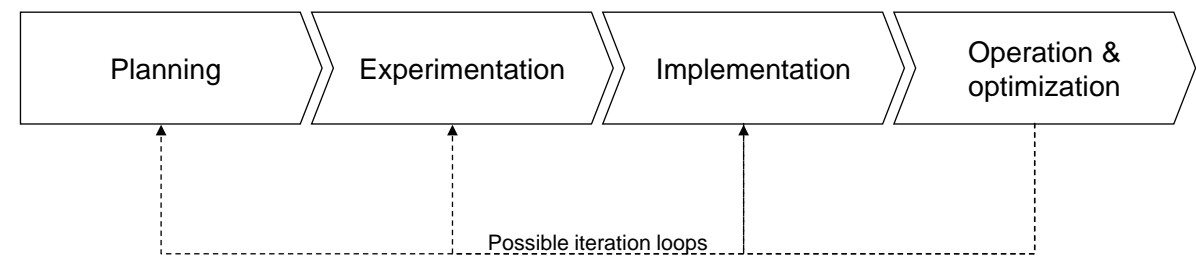

Figure 2. Phase model for building and operating AI systems

\subsection{Planning - Defining the Right Problem to Solve}

The main goal of the planning phase of an AI system is to define the specific problem that should be solved and correspondingly the benefit of the AI system. Therefore, the PEAS framework from Russell et al. [8] is used as a foundation, consisting of four dimensions - performance measure, environment, actuators, and sensors. We generalize the framework for our definition of AI systems by using the four dimensions - performance, environment, measures, and data (PEMD). The reason for the change of the terms actuator and sensor to generic measures and data can be explained by the definition of the different data sources in Section 4.2, as an AI system does not necessarily have to use physical actuators or sensors as interacting objects but can also be based on information systems or databases.

The performance of an AI system is not defined by the evaluation metric of a model (e.g. F1-score in classification or mean squared error in regression) but by the specific performance indicator of the process or problem defined above. Therefore, we differentiate between the model metric for the data-driven model and the system metric for the evaluation or performance metric of the AI system. Whereas the model metrics such as for classification or regression models are well defined, the system metrics are often mentioned but not specified and measured. Furthermore, the relationship of the model metrics and the system metrics are often unclear. The dimension "performance" of the PEMD framework defines the model metrics as well as the system metrics. A typical model metric in binary classification is called recall, which indicates the probability that a positive object is correctly classified as positive. A system metric can be derived from the defined requirements of the AI system. The system metrics are specific business goals such as minimizing costs in manufacturing or increase product quality. By disaggregating the business goal into specific targets, the required models and agents can be derived. The system metric in industrial applications can vary depending on the use case. A typical system metric could be to reduce the energy costs 
of a manufacturing process. One of the possibilities is to detect load peaks of the process with a classification model and react by smoothing the electrical load using different measures which must be defined in the PEMD dimension "measures". Another wellknown system metric in manufacturing goal could be to reduce the number of rejected goods. The goal of the AI system is to maximize or minimize the system metric depending on the problem it solves.

Additionally, the environment of the problem must be determined. The environment can be defined as information systems, processes and physical objects that interact with the AI system. A deep understanding of the environment and its processes is also regarded as domain expertise or knowledge in the field of data science. Especially in industrial applications, a multitude of information systems, high degrees of automation and complex dependencies possess challenges that must also be considered when planning AI systems. For further information on (AI-independent) process and system analysis we refer to [37].

The dimension measures determines the different actions that one or more agents of the AI system can take to achieve the defined goals and to solve the overall problem. Possible actions must be identified. The agents in industrial applications can take the general measures described in section 4.3. Measures for an industrial application could be generating reports with detected anomalies (generation), regulating variables like the engine speed (action) or giving decision support during production planning (decision). It is important to identify these measures without considering the manifestation of the agents, which can be determined afterwards. The performance of the measures is loosely coupled to the overall performance metric. Therefore, the system metric defined in the dimension "performance" can be combined with the different actions defined in the measure dimension. The measure "turn off machine A" is connected to the system metric "minimizing energy consumption of machine A".

The last dimension data defines the sources of information and data that captures the defined environment. It is necessary to determine all possible sources of data like information systems, sensors and actuators as well as external data like weather, customer, or market data. These data sources are described in a data source definition that gives an overview about all the data available, the type of data and how to get access to the data. After the definition of the data sources it is mandatory to model the different data flow processes and the relationships between the data sources. At the end of the planning phase and after determining the performance, environment, measures, and the data a first draft of the AI system can be developed considering our conceptual model for AI systems depicted in section 4.

\subsection{Experimentation - Tracking Experiments for the Best Model}

The goal of the experimentation phase is to find the best possible data-driven models (section 4.3) in an iterative process based on the previously defined and available performance measure and data in the PEMD framework (section 5.2). Therefore, parts of the classic CRISP-DM [15] or other approaches can be used [17]. Existing frameworks do not mention an end-to-end experiment tracking and logging. Tools such as MLFlow [24] track specific model parameters but not the whole process from data 
source to agent. Most of the frameworks and tools for model tracking start their logging when compiling or fitting the model to a specific dataset [24, 25]. Nevertheless, the process of data ingestion, preprocessing and feature engineering and selection from data source to evaluating the model performance has to be tracked and optimized. Afterwards a deep analysis of the process or pipelines is possible where e.g. different feature sets can be compared and analyzed. Does the integration of weather data increase the model performance metric? Is there a difference in performance between scaling to unit variance (standard scaler) and scaling from zero to one (min-max scaler)? To generalize the approaches in model and data tracking we define an experiment as a process with different data processing steps such as data ingestion, data preprocessing, modeling, and evaluation. The granularity of an experiment can vary from simple changes during data preprocessing to more complex hyperparameter tuning. The experiment can be seen as a controlled setup with controllable parameters such as the model architecture but also the used preprocessing steps. For an effective design of experiments we refer to [38, 39]. The parameters of the whole AI system from the data sources used to the evaluation metrics, are referred to as experiment metadata.

In our AI system, an experimental database tracks all metadata during an experiment. A categorization of the metadata that depends on the AI system must be implemented. Experiments should be tracked in a log file or other available approaches to compare different settings. The comparison of experiments and its metadata is the foundation of further optimizing the AI system. Therefore, the PEMD framework for the specific use case can be adjusted when new hypothesis of other influential factors as features are derived. After more research about the overall performance a possible influential factor can be identified (e.g. sensors for measuring the temperature of a process). The new influential factor must be included into the PEMD framework by extending the data dimension. In the initial experimentation phase, after tuning the controllable parameters such as the preprocessing steps and the model architecture the best pipeline depending on the overall performance is chosen. Additionally, during active operation of the AI system the experimentation phase can always be triggered when new experiments are necessary (e.g. new data, modeling technique, processing steps). This also includes the planning phase by extending the specific PEMD framework of the use case.

\subsection{Implementation - Software Engineering for AI Systems}

After experimenting and identifying a suitable model with satisfactory evaluation metrics, the next phase is to implement the trained model into the AI system that was conceptualized in the planning phase. Therefore, it is necessary to choose the right methods and frameworks to build the system on.

As described in Sculley et al. [13] and Lwakatare et al. [23] the software engineering of AI systems has different challenges compared to traditional software engineering. Especially the written software code for data-driven model consists of few lines of code and is just a minor part of the whole software engineering process. The rest of the system consists of configuration, automation, data collection, data verification, testing and troubleshooting, resource management, model analysis, process and metadata management, deployment infrastructure, and monitoring [13]. One of the biggest 
efforts especially in manufacturing is the integration of different data sources into the AI system. Depending on the structure and the access of the data source, application programming interfaces to the defined data sources must be implemented. An information system with a REST API is easier to implement than an old industrial oven without any PLC or smart device that can publish data. The conceptualized and optimized DPUs, derived from the planning and experimentation phase, are used in the implementation phase. Furthermore, it is necessary to implement the right interfaces when agents interact with other information systems or objects such as the energy management system or actuators. The interaction of agents and data-driven models requires inference modules. Three different types of inference modules are possible as micro-services with a REST API to provide online inference, as a model embedded in an edge or mobile device, or as part of a batch inference system.

After implementing and testing the model, the integration with the previous data processing units, model outputs can be evaluated and compared to the experimentation phase. For this, the data flow from the data source is used. If the agent is interacting with other information systems like the energy management system or a MES the engineering of an interface between the agent and the information system is required.

\subsection{Operation \& Optimization - There is no Closing Time for Optimization}

The main goal of the optimization phase is to reassess whether the implemented AI system supports to reach the overall performance goal of solving the specified problem in the planning phase. Therefore, it is necessary to design and plan an AI system management and monitoring functionality for the AI system. To continuously improve the AI system the optimization phase is highly connected to the other phases. The main components to be considered are the monitoring and management of all parameters and metrics defined as experiment metadata in the experimentation phase and the evaluation of the overall defined performance measure from the PEMD framework.

Additionally, the defined processes in the optimization phase should trigger events that start a new iterative cycle of manual or automated experiments. Thus, the processes and the pipeline of the AI system must be monitored and evaluated. Like in the other sectors, we make a distinction between the monitoring and diagnostics of the performance measurement (e.g. energy costs) defined in the PEMD framework and the evaluation metrics (e.g. forecast evaluation metrics) of the implemented models. Furthermore, the inference metrics must be monitored as well. When the time for predicting a value and taking an action is longer than expected an event can be triggered to analyze the problem in the experimentation phase. A solution with visualization methods and automated alerts can help to identify performance issues.

\section{An Anomaly Detection Use Case in Energy Consumption}

For demonstration purpose we applied our definition of an AI system and our developed process in a real-world use case. We developed an AI system to detect anomalies in the electricity energy consumption of a German metal processing company on an 
aggregated (electricity grid connection) and appliance (laser punching machine) level. As manufacturing companies are facing the challenge of simultaneously producing at low cost, complying with environmental regulations, and reducing $\mathrm{CO} 2$ emissions while aiming for a cleaner production, energy consumption must be reduced, and energy efficiency enhanced [40]. In this context, the detection of anomalies in energy consumption is a promising option because anomalies leading to higher energy consumption than necessary, e.g. in poorly maintained, outdated or incorrectly controlled systems, can be detected and countermeasures taken. Our AI system features two smart electricity meters (aggregated and appliance level) integrated via a cloud interface, several DPUs for pre-processing and a long-short term memory based autoencoder (LSTM-AE) to detect anomalies in the time series of energy consumption, which feed an alert system and a visualization dashboard. Using the LSTM-AE offers the advantage of not requiring labelled data and can therefore be easily integrated into the surrounding information systems. The AI system's conceptual model is displayed in Figure 3. The system was gradually developed, implemented, and operated according to the process introduced in Section 5.

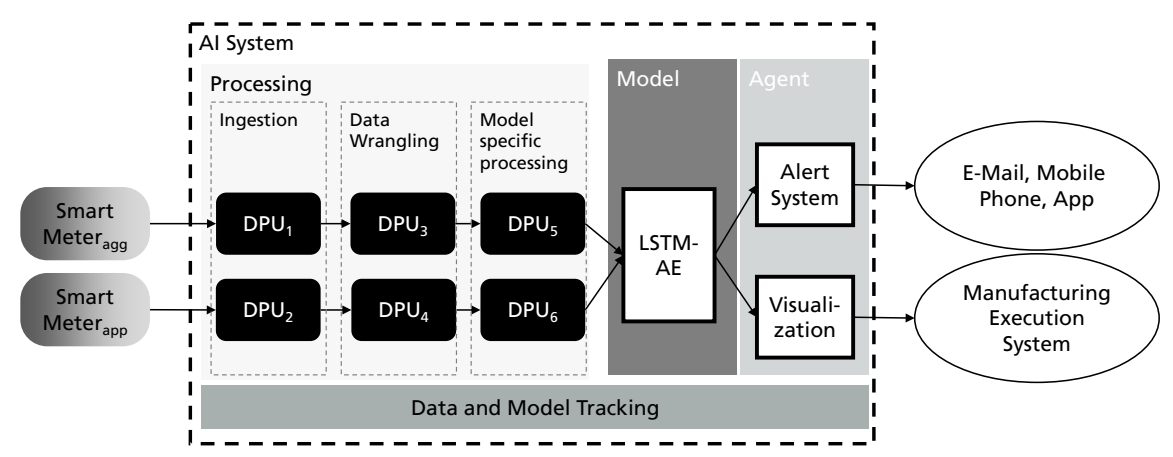

Figure 3. Proposed AI system for anomaly detection

\section{Discussion}

Our presented use case allows us to validate and discuss the developed artifacts with findings from the conducted expert interviews. Since it is suitable here, we combine the discussion and validation with limitations and prospects for further research. With our use case we show that it is possible to build an AI system in practice supported by our artifacts. We realize that our current process does not cover economic aspects and that the success and quality level that can be achieved with the AI system is still to some extent uncertain during the planning phase. Therefore, further aspects from a project management and decision-making perspective could be added to our four-stage process in the planning phase. Within our conducted interviews we found further aspects to be addressed specifically for the manufacturing industry. First, the latency of data streams is very critical (e.g. milliseconds for machine control) and thus must be considered when designing an AI system. Learning on historical data and fixed datasets as typically done in CRISP-DM or KDD might here not be suitable, as the design of a system which 
uses AI must be set in its dynamic environment. The same holds true for approaches in literature that suggest software or software frameworks for machine learning as the superordinate context is often still missing/unclear. Second, safety and reliability of AI systems is very important in manufacturing and must be considered in the planning phase. The modular structure supports this by making e.g. threat modelling easily applicable. Third, interoperability and different levels of automation with different interfaces require generic modeling of systems (= systems engineering) without going into technical details. This supports the statements of all experts with a manufacturing background that one cannot assume that the manufacturing industry has software and machine learning experts, which in turn reinforces our generic and interdisciplinary approach. Fourth, even if everyone talks about industry 4.0 and digital transformation, it actually looks less widespread in reality especially in small and medium-sized companies. Hence, one could even think about of integrating our artifacts in a holistic industry 4.0 roadmap or a digital transformation strategy. Last, some experts mentioned the integrative character of the PEMD framework within the planning phase as well as the integration of agents in the IS ecosystem positively, as e.g. "forecasting of consumption or events seems interesting, but how can I use it in further applications". Summarizing, the acceptance criteria completeness, understandability, and traceability introduced in Section 3 could be fulfilled as far as possible, with prospects for further research derived from minor limitations.

\section{Conclusion}

In our study, we faced the research questions which components are part of an AI system and how a process for planning, implementation, and optimization should be structured. We developed a generic conceptual model of an AI system for use in manufacturing systems and a four-phase model to guide developers and project managers in its realization following a design science research approach. With both research artifacts, we close the knowledge gap of a uniform understanding of components and functionalities of an AI system as well as a structured process for their planning, implementation, and optimization.

Specifically, with our conceptual model for AI systems we depict the central components, structure them, and reveal their relationships. Based on the conceptual model the four-phase model for developing and operating AI systems covers the stages planning, experimentation, implementation, and operation with the possibility to run through iteration loops. Within the planning phase, we provide a structured procedure with our PEMD framework with which the dimensions performance, environment, measures, and data are determined. In the experimentation phase, existing methods such as CRISP-DM can be applied. The implementation phase focuses on the software realization before the operation \& optimization covers the processes of the AI system's optimization in operation.

Our developed artifacts have several implications and benefits. The conceptual model as well as the phase model are designed non-technical, which means they are easy to understand and provide a common discussion basis and tool for developers, 
engineers, data scientists, and managers. This makes the introduction of AI systems easier in practice because there is a clear understanding and a structured procedure for all stakeholders involved. In addition, our phase model is independent of existing frameworks and acts as a meta-model that allows the integration of existing models and frameworks on a more detailed level. Furthermore, our conceptual and phase model is technology-independent and thus enables to draw on the full potential and at the same time, it prevents bias due to possible technological limitations.

\section{References}

1. Leo Kumar, S.P.: State of The Art-Intense Review on Artificial Intelligence Systems Application in Process Planning and Manufacturing. Engineering Applications of Artificial Intelligence 65, 294-329 (2017)

2. Lee, J., Davari, H., Singh, J., Pandhare, V.: Industrial Artificial Intelligence for industry 4.0-based manufacturing systems. Manufacturing Letters 18, 20-23 (2018)

3. Monostori, L., Markus, A., van Brussel, H., Westkämpfer, E.: Machine Learning Approaches to Manufacturing. CIRP Annals 45, 675-712 (1996)

4. Patel, A.R., Ramaiya, K.K., Bhatia, C.V., Shah, H.N., Bhavsar, S.N.: Artificial Intelligence: Prospect in Mechanical Engineering Field—A Review. In: Kotecha, K., Piuri, V., Shah, H.N., Patel, R. (eds.) Data Science and Intelligent Applications, 52, pp. 267-282. Springer Singapore, Singapore (2021)

5. Wuest, T., Weimer, D., Irgens, C., Thoben, K.-D.: Machine learning in manufacturing: advantages, challenges, and applications. Production \& Manufacturing Research 4, 23-45 (2016)

6. Capgemini: Scaling AI in Manufacturing Operations: A Practitioners' Perspective (2019)

7. Mehrabi, M., Ulsoy, A., Koren, Y.: Reconfigurable manufacturing systems: Key to future manufacturing. Kluwer Academic Publishers, Boston (2000)

8. Russell, S.J., Norvig, P., Davis, E., Edwards, D.: Artificial intelligence. A modern approach. Pearson, Boston, Columbus, Indianapolis, New York, San Francisco, Upper Saddle River, Amsterdam, Cape Town, Dubai, London, Madrid, Milan, Munich, Paris, Montreal, Toronto, Delhi, Mexico City, Sao Paulo, Sydney, Hong Kong, Seoul, Singapore, Taipei, Tokyo (2016)

9. Nalchigar, S., Yu, E.: Conceptual Modeling for Business Analytics: A Framework and Potential Benefits. In: 2017 IEEE 19th Conference on Business Informatics (CBI), pp. 369-378. IEEE (2017 - 2017)

10. Wang, S., Wan, J., Zhang, D., Di Li, Zhang, C.: Towards smart factory for industry 4.0: a self-organized multi-agent system with big data based feedback and coordination. Computer Networks 101, 158-168 (2016)

11. van den Heuvel, W.-J., Tamburri, D.A.: Model-Driven ML-Ops for Intelligent Enterprise Applications: Vision, Approaches and Challenges. In: Shishkov, B. (ed.) Business Modeling and Software Design, 391, pp. 169-181. Springer International Publishing, Cham (2020) 
12. Simard, P.Y., Amershi, S., David M. Chickering, Alicia Edelman Pelton, Soroush Ghorashi, Christopher Meek, Gonzalo Ramos, Jina Suh, Johan Verwey, Mo Wang, et al.: Machine Teaching A New Paradigm for Building Machine Learning Systems (2017)

13. D. Sculley, Gary Holt, Daniel Golovin, Eugene Davydov, Todd Phillips, Dietmar Ebner, Vinay Chaudhary, Michael Young, Jean-François Crespo, Dan Dennison: Hidden Technical Debt in Machine Learning Systems. Advances in Neural Information Processing Systems (2015)

14. Fayyad, Usama, Haussler, David, Stolorz, E, P.: KDD for Science Data Analysis: Issues and Examples (1996)

15. Wirth, R., Hipp, J.: CRISP-DM: Towards a Standard Process Model for Data Mining (2000)

16. Microsoft: Team Data Science Process Documentation. Learn how to use the Team Data Science Process, an agile, iterative data science methodology for predictive analytics solutions and intelligent applications., https://docs.microsoft.com/en-us/azure/machine-learning/team-data-scienceprocess/

17. Kessler, R.: Towards a Cross-Company Data and Model Platform for SMEs. In: Abramowicz, W., Corchuelo, R. (eds.) Business Information Systems Workshops, 373, pp. 661-671. Springer International Publishing, Cham (2019)

18. Colomo-Palacios, R.: Towards a Software Engineering Framework for the Design, Construction and Deployment of Machine Learning-Based Solutions in Digitalization Processes. In: Visvizi, A. (ed.) Research \& Innovation Forum 2019. Technology, innovation, education, and their social impact, pp. 343-349. Springer, Cham (op. 2019)

19. Amershi, S., Begel, A., Bird, C., DeLine, R., Gall, H., Kamar, E., Nagappan, N., Nushi, B., Zimmermann, T.: Software Engineering for Machine Learning: A Case Study. IEEE/ACM 41st International Conference on Software Engineering: Software Engineering in Practice, 291-300 (2019)

20. Kessler, R., Gómez, J.M.: Implikationen von Machine Learning auf das Datenmanagement in Unternehmen. HMD 57, 89-105 (2020)

21. Moreb, M., Mohammed, T.A., Bayat, O.: A Novel Software Engineering Approach Toward Using Machine Learning for Improving the Efficiency of Health Systems. IEEE Access 8, 23169-23178 (2020)

22. Kriens, P., Verbelen, T.: Software Engineering Practices for Machine Learning (2019)

23. Lwakatare, L.E., Raj, A., Bosch, J., Olsson, H.H., Crnkovic, I.: A Taxonomy of Software Engineering Challenges for Machine Learning Systems: An Empirical Investigation. In: Kruchten, P., Fraser, S., Coallier, F. (eds.) Agile Processes in Software Engineering and Extreme Programming, 355, pp. 227-243. Springer International Publishing, Cham (2019)

24. Chen, A., Chow, A., Davidson, A., DCunha, A., Ghodsi, A., Hong, S.A., Konwinski, A., Mewald, C., Murching, S., Nykodym, T., et al.: Developments in MLflow. Proceedings of the Fourth International Workshop on Data Management for End-to-End Machine Learning, 1-4 (2020) 
25. Baylor, D., Breck, E., Cheng, H.-T., Fiedel, N., Foo, C.Y., Haque, Z., Haykal, S., Ispir, M., Jain, V., Koc, L., et al.: TFX - A TensorFlow-Based Production-Scale Machine Learning Platform, 1387-1395 (2017)

26. Hevner, March, Park, Ram: Design Science in Information Systems Research. MIS Quarterly 28, 75 (2004)

27. Gregor, S., Hevner, A.R.: Positioning and Presenting Design Science Research for Maximum Impact. MIS Quarterly 37, 337-355 (2013)

28. Wand, Y., Monarchi, D.E., Parsons, J., Woo, C.C.: Theoretical foundations for conceptual modelling in information systems development. Decision Support Systems 15, 285-304 (1995)

29. Brodie, M.L. (ed.): On conceptual modelling. Perspectives from artificial intelligence, databases, and programming languages. Springer, Berlin (1986)

30. Rishe, N.: Database design. The semantic modeling approach. McGraw-Hill, New York (1992)

31. Felderer, M., Reussner, R., Rumpe, B.: Software Engineering und SoftwareEngineering-Forschung im Zeitalter der Digitalisierung. Informatik Spektrum (2020)

32. Selcuk, S.: Predictive maintenance, its implementation and latest trends. Proceedings of the Institution of Mechanical Engineers, Part B: Journal of Engineering Manufacture 231, 1670-1679 (2017)

33. Maass, W., Parsons, J., Purao, S., Storey, V.C., Woo, C.: Data-Driven Meets Theory-Driven Research in the Era of Big Data: Opportunities and Challenges for Information Systems Research. JAIS, 1253-1273 (2018)

34. Kohne, T., Ranzau, H., Panten, N., Weigold, M.: Comparative study of algorithms for optimized control of industrial energy supply systems. Energy Inform 3 (2020)

35. Khan, A., Turowski, K.: A Survey of Current Challenges in Manufacturing Industry and Preparation for Industry 4.0. Proceedings of the First International Scientific Conference 'Intelligent Information Technologies for Industry' (IITI'16), 15-26 (2016)

36. Hofmann, P., Jan Jöhnk, Dominik Protschky, Nils Urbach: Developing Purposeful AI Use Cases : A Structured Method and Its Application in Project Management. In: Entwicklungen, Chancen und Herausforderungen der Digitalisierung. Band 1.Proceedings der 15. Internationalen Tagung Wirtschaftsinformatik 2020, pp. 33-49. GITO Verlag, Berlin (2020)

37. Krallmann, H. (ed.): Systemanalyse im Unternehmen. Prozessorientierte Methoden der Wirtschaftsinformatik. Oldenbourg, München (2007)

38. Stanley, J.C.: The Influence of Fisher's “The Design of Experiments” on Educational Research Thirty Years Later. American Educational Research Journal 3, 223-229 (1966)

39. Box, J.F.: R. A. Fisher and the Design of Experiments, 1922-1926. The American Statistician 34, 1 (1980)

40. Bauer, D., Hieronymus, A., Kaymakci, C., Köberlein, J., Schimmelpfennig, J., Wenninger, S., Zeiser, R.: Wie IT die Energieflexibilitätsvermarktung von Industrieunternehmen ermöglicht und die Energiewende unterstützt. HMD (2020) 